\title{
Local smoothing results for the Ricci flow in dimensions two and three
}

\author{
Miles Simon
}

\begin{abstract}
We present local estimates for solutions to the Ricci flow, without the assumption that the solution has bounded curvature. These estimates lead to a generalisation of one of the pseudolocality results of G Perelman in dimension two.
\end{abstract}

35B65, 53C44

\section{Introduction}

In this paper, unless otherwise specified, a solution $(M, g(t))_{t \in[0, T)}$ to Ricci flow refers to a family $(M, g(t))_{t \in[0, T)}$ of smooth (in space and time) Riemannian manifolds which are complete for all $t \in[0, T)$, solve $(\partial / \partial t) g(t)=-2 \operatorname{Ric}(g(t))$ and have no boundary. We do not require (unless otherwise stated) that the solution has bounded curvature.

In the paper [12], G Perelman proved the following fact: if a ball ${ }^{0} B_{r}\left(x_{0}\right)$ in $(M, g(0))$ at time zero is almost Euclidean, and $(M, g(t))_{t \in[0, T)}$ is a solution to the Ricci flow with bounded curvature, then for small times $t \in[0, \epsilon(n, r))$, we have estimates on how the curvature behaves on balls ${ }^{t} B_{\epsilon r}\left(x_{0}\right)$. There are a number of versions of his theorem; see [12, Theorems 10.1 and 10.3] for proofs and the definitions of almost Euclidean. See Chau, Tam and Yu [3], Lu [11], Chow et al [6], Kleiner and Lott [10] Wang [16] and Chen [4] for alternative proofs and related results. In dimension two, we show that a similar result holds under weaker initial assumptions.

Theorem 1.1 Let $1>\sigma, \alpha>0, v_{0}, r>0, N>1$ be given. Let $\left(M^{2}, g(t)\right)_{t \in[0, T)}$ be a smooth complete solution to Ricci flow, $x_{0} \in M$, and assume that

- $\quad \operatorname{vol}^{0} B_{r}\left(x_{0}\right) \geq v_{0} r^{2}$,

- $\mathrm{R}(g(0)) \geq-\frac{N}{r^{2}}$ on ${ }^{0} B_{r}\left(x_{0}\right)$. 
Then there exists a $\widetilde{v}_{0}=\widetilde{v}_{0}\left(v_{0}, \sigma, N, \alpha\right)>0$ and a $\delta_{0}=\delta_{0}\left(v_{0}, \sigma, N, \alpha\right)>0$ such that

- $\operatorname{vol}\left({ }^{t} B_{r(1-\sigma)}\left(x_{0}\right)\right) \geq \tilde{v}_{0} r^{2}$,

- $\mathrm{R}(g(t)) \geq-\frac{(N+\alpha)}{r^{2}}$ on ${ }^{t} B_{r(1-\sigma)}\left(x_{0}\right)$,

- $|\mathrm{R}(g(t))| \leq \frac{1}{\delta_{0}^{2} t}$ on ${ }^{t} B_{r(1-\sigma)}\left(x_{0}\right)$,

as long as $t \leq\left(\delta_{0}\right)^{2} r^{2}$ and $t \in[0, T)$.

Remark 1.2 Notice that we do not require that a region be almost Euclidean here (see [12, Theorems 10.1 and 10.3] for the definition of almost Euclidean). If the ball ${ }^{0} B_{1}\left(x_{0}\right)$ is almost cone like (that is, it is as close as we like in the Gromov-Hausdorff sense to an Euclidean cone and has $\mathrm{R} \geq-2$ ) then the Theorem (with $r=1$ ) still applies. This means that the interior of regions which are cone like in this sense will be smoothed out by Ricci flow in two dimensions, regardless of what the solution looks like outside of this region. Both of G Perelman's [12, Theorems 10.1 and 10.3] do not apply to this situation.

Remark 1.3 By scaling, it suffices to prove the Theorem for $r=1$.

Remark 1.4 In the estimate $|\operatorname{Riem}(g(t))| \leq \frac{1}{\delta_{0} t}$, it is not possible to improve the constant $\delta_{0}$ to an arbitrary constant $\delta_{0}>0$ for a short time. This is because, solutions coming out of nonnegatively curved cones exist which have curvature behaviour immediately like $\frac{c}{t}$ where $c>0$ depends on the cone angle (see Schulze and the author [13]). In G Perelman's first pseudolocality result [12, Theorem 10.1], where he assumes that a ball $B_{r}\left(y_{0}\right)$ at time zero is almost Euclidean, he showed that it is possible to obtain an estimate of the form $|\operatorname{Riem}(g(t))| \leq \frac{\alpha}{t}$ on a smaller ball for arbitrary $\alpha$ at least for some short time interval depending on $\alpha$, as long as the initial ball is close enough to the Euclidean ball. Here close enough means, that $(\operatorname{vol}(\partial \Omega))^{n} \geq(1-\delta) c_{n}(\operatorname{vol}(\Omega))^{n-1}$ for any $\Omega \subset B_{r}\left(y_{0}\right)$, where $c_{n}$ is the Euclidean isoperimetric constant, $\mathrm{R} \geq-\frac{1}{r^{2}}$ and $\delta=\delta(n, \alpha)>0$ is small enough.

The second theorem is valid in three dimensions. In contrast to the above theorem, we need to have information on how the curvature is behaving (in time) in the balls we are considering in order to draw (stronger) conclusions.

Theorem 1.5 Let $r, v_{0}>0, N>1,1>\sigma>0, V>0$ be given. Let $\left(M^{3}, g(t)\right)_{t \in[0, T)}$ be a smooth complete solution to Ricci flow with $T \leq 1$ and let $x_{0} \in M$ be a point such that 
- $\operatorname{vol}{ }^{0} B_{r}\left(x_{0}\right) \geq v_{0} r^{3}$,

- $\mathcal{R}(g(0)) \geq-\frac{V}{r^{2}}$ on ${ }^{0} B_{r}\left(x_{0}\right)$,

- $|\operatorname{Riem}(g(t))| \leq \frac{N}{t}$ on ${ }^{t} B_{r}\left(x_{0}\right)$, for all $t \in(0, T)$.

Then there exists a $\widetilde{v}_{0}=\widetilde{v}_{0}\left(v_{0}, N, \sigma, V\right)>0$ and a $\delta_{0}=\delta_{0}\left(v_{0}, N, \sigma, V\right)>0$ such that

- $\operatorname{vol}\left({ }^{t} B_{r}\left(x_{0}\right)\right) \geq \widetilde{v}_{0} r^{3}$,

- $\mathcal{R}(g(t)) \geq-\frac{400 N V}{r^{2}}$ on ${ }^{t} B_{r(1-\sigma)}\left(x_{0}\right)$,

as long as $t \leq r^{2}\left(\delta_{0}\right)^{2}$ and $t \in[0, T)$.

Remark 1.6 By scaling arguments it suffices to prove the theorem for $r=1$ and $V=\frac{1}{400 N}$; see Remark 5.3.

Remark 1.7 As in the two-dimensional case (Theorem 1.1 above), the regions which are considered are not necessarily almost Euclidean at time zero.

Remark 1.8 Related results were proved recently in a preprint by Chen, Xu and Zhang [5]. There the authors require that the curvature of the solution be uniformly bounded by a constant $c$ on a ball for the times $t \in[0, S)$ being considered; see [5].

Remark 1.9 The above results were first presented in November 2011 at the H.I.M. workshop "Geometric Flows" in Bonn.

Acknowledgements We thank Robert Haslhofer for comments on and discussions about an earlier version of this paper.

\section{A local bound for the curvature on regions whose curva- ture is bounded from below}

We use the following notation in this paper.

Notation - $d(x, y, t)=d_{t}(x, y)=d(g(t))(x, y)$ is the distance from $x$ to $y$ in $M$ with respect to the metric $g(t)$;

- $d_{t}(x)=d_{t}\left(x, x_{0}\right)$ is distance from $x$ to $x_{0}$ with respect to the metric $g(t)$ for some fixed $x_{0}$; 
- ${ }^{t} B_{r}(x):=$ ball of radius $r>0$, centre point $x \in M$ measured with respect to $(M, g(t))$, that is ${ }^{t} B_{r}(x):=\left\{y \in M \mid d_{t}(x, y)<r\right\}$;

- $\operatorname{vol}\left({ }^{t} B_{r}(x)\right):=$ volume of ${ }^{t} B_{r}(x)$ with respect to the volume form $d \mu_{t}$ induced by $g(t)$;

- $\operatorname{Riem}(g(t))(x)=\operatorname{Riem}(x, t)$ is the Riemannian curvature tensor of the metric $g(t)$ at the point $x \in M$;

- $\mathcal{R}(g(t))(x):=\mathcal{R}(x, t)$ is the curvature operator of $(M, g(t))$ at the point $x \in M$ : $\mathcal{R}(x, t)(V, W):=\operatorname{Riem}_{i j k l}(x, t) V^{i j} W^{k l}$ for 2 -forms $V, W\left(\operatorname{Riem}_{i j k l}\right.$ is the curvature tensor of $g(t)$ in local coordinates, and we have $V=V_{i j} d x^{i} \otimes d x^{j}$, $W=W_{i j} d x^{i} \otimes d x^{j}$, and $\left.V^{k m}=V_{i j} g^{k i} g^{m j}, W^{k m}=W_{i j} g^{k i} g^{m j}\right)$;

- $\mathrm{R}(x, t)$ is the scalar curvature of $(M, g(t))$ at the point $x \in M$.

Let $(M, g(t))_{t \in[0, T]}, T \leq 1$ be a smooth complete solution to Ricci flow. We wish to prove estimates on a ball of radius $r$ at time $t \in[0, T]$, assuming the curvature operator stays bounded from below on ${ }^{t} B_{r}(x)$ for all $t \in[0, T]$ and the volume of ${ }^{t} B_{r}(x)$ is bounded from below for all $t \in[0, T]$. The estimates will depend on $n, r$ and the bounds from below. A local result of this type was obtained by B-L Chen in the proof of [4, Theorem 3.6], under the assumption that the curvature operator is nonnegative on all of $(M, g(t))_{t \in[0, T)}$. A global result of this type was obtained by the author; see both [14, Lemma 2.4] and and [15, Lemma 4.3].

In the proof of of [4, Theorem 3.6] by B-L Chen, the author uses a point picking argument of $\mathrm{G}$ Perelman before rescaling to obtain a contradiction to [12, Proposition 11.4] (in the proof of [14, Lemma 2.4] and [15, Lemma 4.3] we used a more global point picking type argument of $\mathrm{R}$ Hamilton and also obtained a contradiction to [12, Proposition 11.4] after scaling). The point picking argument of G Perelman is more suited to this local situation, and so we use it in the following.

The proof follows the lines given in the proof of [4, Theorem 3.6]. A number of modifications are necessary.

Theorem 2.1 Let $r, v_{0}>0,1>\sigma>0$ and $\left(M^{n}, g(t)\right)_{t \in[0, T)}$ be a smooth complete solution to Ricci-flow which satisfies

(a) $\operatorname{vol}\left({ }^{t} B_{r}\left(x_{0}\right)\right) \geq v_{0} r^{n}$,

(b) $\mathcal{R}(x, t) \geq-\frac{1}{r^{2}}$ for all $t \in[0, T), x \in{ }^{t} B_{r}\left(x_{0}\right)$.

Then, there exists a $N=N\left(n, v_{0}, \sigma\right)<\infty$ such that

(c) $\mid$ Riem $\mid \leq \frac{N^{2}}{t}+\frac{N^{2}}{r^{2}}$ for all $x \in{ }^{t} B_{r(1-\sigma)}\left(x_{0}\right), t \in\left[0, \frac{1}{r^{2} N^{2}}\right) \cap[0, T)$. 
Proof By scaling, it suffices to prove the case $r=1$. Assume that the statement is false. Then we can find solutions $\left(M_{i}^{n}, g_{i}(t)\right)_{t \in\left[0, T_{i}\right)}, T_{i} \leq 1, i \in \mathbb{N},(i \neq 0$ for notational reasons: we shall use the symbol $x_{0}$ in a moment) and points $x_{i} \in M_{i}$ such that

(a) $\operatorname{vol}\left(B_{1}\left(x_{i}, t\right)\right) \geq v_{0}$ for all $t \in\left[0, T_{i}\right)$,

(b) $\mathcal{R}(x, t) \geq-1$ for all $t \in\left[0, T_{i}\right), x \in{ }^{t} B_{1}\left(x_{i}\right)$,

and points $t_{i} \in\left[0, T_{i}\right), z_{i} \in{ }^{t_{i}} B_{(1-\sigma)}\left(x_{i}\right)$ such that $t_{i} \leq \frac{1}{N_{i}^{2}}$ and

$$
\left|\operatorname{Riem}\left(z_{i}, t_{i}\right)\right| \geq \frac{N_{i}^{2}}{t_{i}}+N_{i}^{2},
$$

with $N_{i} \rightarrow \infty$ as $i \rightarrow \infty$. Fix $i \in \mathbb{N}$ for the moment and define $M:=M_{i}$, $x_{0}:=x_{i}, s_{0}:=t_{i}, g(t):=g_{i}(t), y_{0}:=z_{i}, T=T_{i}, d_{t}(x):=\operatorname{dist}\left(g_{i}(t)\right)\left(x, x_{i}\right)=$ $\operatorname{dist}(g(t))\left(x, x_{0}\right), A=\left(\sigma N_{i}\right) / 8, \epsilon:=1 / N_{i}$ and $\alpha=N_{i}^{2}$. Then $A \epsilon \leq \sigma / 8$ and $s_{0} \leq \epsilon^{2}$ and $g$ solves RF on $[0, T]$ with $T \leq \epsilon^{2}$ and $|\operatorname{Riem}|\left(y_{0}, s_{0}\right) \geq \alpha / s_{0}+1 / \epsilon^{2}$. That is, we are in the setup of [12, Theorem 10.1, Claim 1], except he requires $g$ be a RF on $\left[0, \epsilon^{2}\right]$. Examining the argument of Perelman, we see that we only need that $g$ solves RF on $[0, T]$ with $T \leq \epsilon^{2}$, as the subsequent point picking argument only looks at times less than or equal to $s_{0}$ (see the proof of [12, Theorem 10.1, Claim 1]). Also, we do not have $d_{s_{0}}\left(y_{0}\right) \leq \epsilon$ : we have $d_{s_{0}}\left(y_{0}\right)=d_{s_{0}}\left(x_{i}, z_{i}\right) \leq(1-\sigma)$. This causes no problem in the point picking argument, and merely leads to the term $2 A \epsilon+1-\sigma$ appearing in place of $2 A \epsilon+\epsilon$ in the estimate (2-1) below. Using [12, Theorem 10.1, Claim 1], we obtain new points $\bar{y}_{0} \in M, \bar{s}_{0}$ satisfying

$$
\begin{gathered}
\bar{s}_{0} \leq s_{0}, \quad d_{\bar{s}_{0}}\left(\bar{y}_{0}\right) \leq 2 A \epsilon+(1-\sigma), \quad(\leq 3) \\
\left|\operatorname{Riem}\left(\bar{y}_{0}, \bar{s}_{0}\right)\right| \geq \frac{\alpha}{\bar{s}_{0}}=\frac{N_{i}^{2}}{\bar{s}_{0}}, \quad\left(\geq N_{i}^{2}\right)
\end{gathered}
$$

and

$$
|\operatorname{Riem}(x, t)| \leq 4\left|\operatorname{Riem}\left(\bar{y}_{0}, \bar{s}_{0}\right)\right|,
$$

whenever $|\operatorname{Riem}(x, t)| \geq \frac{\alpha}{t}, t \leq \bar{s}_{0}\left(\leq s_{0}\right)$ and $d_{t}(x) \leq d_{\bar{s}_{0}}\left(\bar{y}_{0}\right)+A|\operatorname{Riem}|^{-1 / 2}\left(\bar{y}_{0}, \bar{s}_{0}\right)$. Hence a version of [12, Theorem 10.1, Claim 2] is applicable. We follow the first part of the argument of B Kleiner and J Lott in the proof of Lemma 32.1 of the arXiv version of their paper [10]. This gives us

$$
|\operatorname{Riem}(x, t)| \leq 4\left|\operatorname{Riem}\left(\bar{y}_{0}, \bar{s}_{0}\right)\right|
$$


whenever

$$
\begin{aligned}
\bar{s}_{0}-(1 / 2) \alpha Q^{-1} & \leq t \leq \bar{s}_{0}, \\
d_{t}(x) & \leq d_{\bar{s}_{0}}\left(\bar{y}_{0}\right)+A Q^{-1 / 2},
\end{aligned}
$$

where here we have $Q:=\operatorname{Riem}\left(\bar{y}_{0}, \bar{s}_{0}\right)$. Note that the second inequality just says that

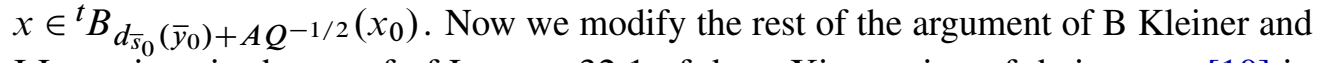
$\mathrm{J}$ Lott given in the proof of Lemma 32.1 of the arXiv version of their paper [10] in order to obtain a product region on which the curvature is bounded. Notice that we do not have $\alpha \leq \frac{1}{100 n}$, and therefore modifications are necessary. We claim that

whenever

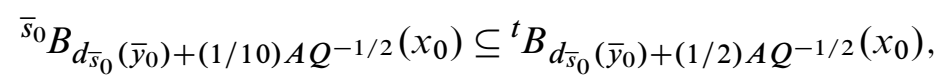

$$
\bar{s}_{0}-M_{0} Q^{-1} \leq t \leq \bar{s}_{0},
$$

where $M_{0}$ is a fixed large constant. We assume in the following that $Q$ and $A$ are large (a lot larger than $M_{0}$ ). Let $x \in{ }^{\bar{s}_{0}} B_{d_{\bar{s}_{0}}}\left(\bar{y}_{0}\right)+(1 / 10) A Q^{-1 / 2}\left(x_{0}\right)$. As long as

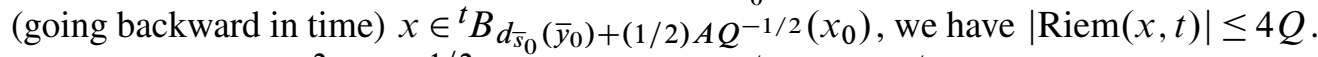
Choose $r=\left(1 / M_{0}^{2}\right) A Q^{-1 / 2}$. Then note that ${ }^{t} B_{r}(x) \subseteq{ }^{t} B_{d_{\bar{s}_{0}}\left(\bar{y}_{0}\right)+A Q^{-1 / 2}\left(x_{0}\right) \text { by }}$ the triangle inequality and hence $|\operatorname{Riem}(\cdot, t)| \leq 4 Q$ on ${ }^{t} B_{r}(x)$ (note that trivially,

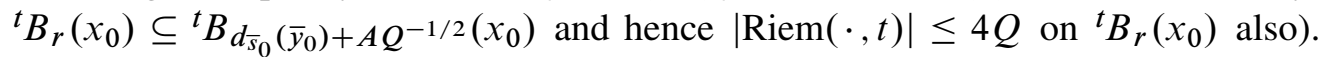
Using [12, Lemma 8.3(b)] we see that

Hence

$$
\frac{\partial}{\partial t} d_{t}\left(x_{0}, x\right)(t) \geq-2(n-1)\left(\frac{2}{3} 4 Q \frac{1}{M_{0}^{2}} A Q^{-1 / 2}+M_{0}^{2} A^{-1} Q^{1 / 2}\right) .
$$

$$
\begin{aligned}
d_{t}\left(x_{0}, x\right)-d_{\bar{s}_{0}}\left(x_{0}, x\right) & \leq M_{0} Q^{-1} 2(n-1)\left(\frac{2}{3} 4 Q \frac{1}{M_{0}^{2}} A Q^{-1 / 2}+M_{0}^{2} A^{-1} Q^{1 / 2}\right) \\
& \leq \frac{8(n-1)}{M_{0}} A Q^{-1 / 2}+2(n-1) M_{0}^{3} A^{-1} Q^{-1 / 2} \\
& \leq \frac{16(n-1)}{M_{0}} A Q^{-1 / 2},
\end{aligned}
$$

where we have used that $A \gg M_{0}$, and $\left|t-\bar{s}_{0}\right| \leq M_{0} Q^{-1}$. That is

$$
\begin{aligned}
d_{t}(x) & \leq d_{\bar{s}_{0}}\left(x_{0}, x\right)+\frac{16(n-1)}{M_{0}} A Q^{-1 / 2} \\
& \leq d_{\bar{s}_{0}}\left(\bar{y}_{0}\right)+\frac{1}{10} A Q^{-1 / 2}+\frac{16(n-1)}{M_{0}} A Q^{-1 / 2} \\
& \leq d_{\bar{s}_{0}}\left(\bar{y}_{0}\right)+\frac{1}{8} A Q^{-1 / 2}
\end{aligned}
$$


and hence $x \in{ }^{t} B_{d_{\bar{s}_{0}}}\left(\bar{y}_{0}\right)+(1 / 8) A Q^{-1 / 2}\left(x_{0}\right)$. Therefore, $x \in{ }^{t} B_{d_{\bar{s}_{0}}}\left(\bar{y}_{0}\right)+(1 / 2) A Q^{-1 / 2}\left(x_{0}\right)$ will not be violated for $\bar{s}_{0}-M_{0} Q^{-1} \leq t \leq \bar{s}_{0}$, as claimed.

Now assume that $d_{\bar{s}_{0}}\left(x, \bar{y}_{0}\right) \leq(1 / 10) A Q^{-1 / 2}$ (ie $\left.x \in{ }^{\bar{s}_{0}} B_{(1 / 10)} A Q^{-1 / 2}\left(\bar{y}_{0}\right)\right)$ and that $\bar{s}_{0}-M_{0} Q^{-1} \leq t \leq \bar{s}_{0}$. The triangle inequality implies that

$$
\begin{aligned}
d_{\bar{s}_{0}}\left(x, x_{0}\right) & \leq d_{\bar{s}_{0}}\left(x, \bar{y}_{0}\right)+d_{\bar{s}_{0}}\left(x_{0}, \bar{y}_{0}\right) \\
& \leq \frac{1}{10} A Q^{-1 / 2}+d_{\bar{s}_{0}}\left(\bar{y}_{0}\right),
\end{aligned}
$$

and hence

$x \in{ }^{\bar{s}_{0}} B_{(1 / 10) A Q^{-1 / 2}}\left(\bar{y}_{0}\right) \subseteq{ }^{\bar{s}_{0}} B_{(1 / 10) A Q^{-1 / 2}+d_{\bar{s}_{0}}\left(\bar{y}_{0}\right)}\left(x_{0}\right) \subseteq{ }^{t} B_{(1 / 2) A Q^{-1 / 2}+d_{\bar{s}_{0}}\left(\bar{y}_{0}\right)}\left(x_{0}\right)$

(as we just showed), so $|\operatorname{Riem}(x, t)| \leq 4 Q$ in view of $(2-3)$. That is $|\operatorname{Riem}(\cdot, t)| \leq 4 Q$

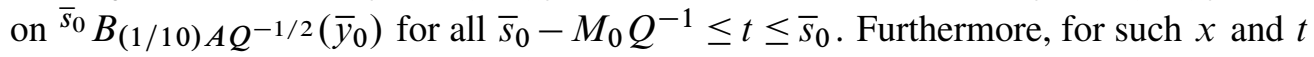
we have $x \in{ }^{t} B_{(1 / 2) A Q^{-1 / 2}+d_{\bar{s}_{0}}\left(\bar{y}_{0}\right)}\left(x_{0}\right)$, as we just showed, and using (2-1), we see that

$$
\begin{aligned}
\frac{1}{2} A Q^{-1 / 2}+d_{\bar{s}_{0}}\left(\bar{y}_{0}\right) & \leq \frac{1}{2} A Q^{-1 / 2}+2 A \epsilon+(1-\sigma) \\
& \leq \frac{\sigma}{4}+\frac{\sigma}{4}+(1-\sigma) \\
& \leq\left(1-\frac{\sigma}{2}\right)
\end{aligned}
$$

which gives us that $x \in{ }^{t} B_{(1-\sigma / 2)}\left(x_{0}\right)$, and there we have that $\mathcal{R} \geq-1$. Note we have used here that $Q \geq N_{i}^{2}$ (follows from the inequality (2-2)) and the definition of $A$ and $\epsilon$. Using the Bishop-Gromov volume comparison principle, we also see that

$$
\operatorname{vol}\left({ }^{t} B_{s}(x)\right) \geq \widetilde{v}\left(\sigma, v_{0}\right) s^{n}
$$

for such $x$ and $t$ and all $s \leq \sigma / 10$ in view of the fact that $\operatorname{vol}\left({ }^{t} B_{1}\left(x_{0}\right)\right) \geq v_{0}$. Taking $x=\bar{y}_{0} \in \bar{s}_{0} B_{(1 / 10) A Q^{-1 / 2}\left(\bar{y}_{0}\right)}$ we get

$$
\operatorname{vol}\left({ }^{t} B_{S}\left(\bar{y}_{0}\right)\right) \geq \widetilde{v}\left(\sigma, v_{0}\right) s^{n}
$$

for all $s \leq \sigma / 10$ and $\bar{s}_{0}-M_{0} Q^{-1} \leq t \leq \bar{s}_{0}$.

Defining $\bar{z}_{i}:=\bar{y}_{0}, \bar{t}_{i}:=\bar{s}_{0}$ and substituting $\alpha=N_{i}$ etc. back into the above we get

$$
\begin{gathered}
|\operatorname{Riem}(x, t)| \leq 4 Q_{i}, \\
\mathcal{R}(x, t) \geq-1,
\end{gathered}
$$


whenever

$$
\begin{aligned}
\overline{t_{i}}-M_{0} Q^{-1} & \leq t \leq \overline{t_{i}}, \\
d_{\bar{t}_{i}}\left(x, \bar{z}_{i}\right) & \leq \frac{1}{10} A_{i} Q_{i}^{-1 / 2},
\end{aligned}
$$

where $Q_{i}:=\left|\operatorname{Riem}\left(\bar{z}_{i}, \bar{t}_{i}\right)\right| \geq N_{i}^{2}$ and $A_{i}=\frac{\sigma N_{i}}{8}$. We also have the volume estimate

$$
\operatorname{vol}\left({ }^{t} B_{s}\left(\bar{z}_{i}\right)\right) \geq \widetilde{v}\left(\sigma, v_{0}\right) s^{n}
$$

for all $s \leq \frac{\sigma}{10}, \bar{t}_{i}-M_{0} Q^{-1} \leq t \leq \bar{t}_{i}$.

Rescaling the solutions by $Q_{i}$ and shifting time by $t_{i}$ we get solutions to Ricci flow with

$$
\begin{aligned}
& |\operatorname{Riem}(x, t)| \leq 4, \\
& \mathcal{R}(x, t) \geq-\frac{1}{Q_{i}},
\end{aligned}
$$

whenever

$$
\begin{gathered}
-M_{0} \leq t \leq 0, \\
d_{0}\left(x, \bar{z}_{i}\right) \leq \frac{\sigma N_{i}}{80},
\end{gathered}
$$

in view of the definition of $A_{i}=\frac{\sigma N_{i}}{8}$. After scaling, the volume estimate is

$$
\operatorname{vol}\left({ }^{t} B_{S}\left(\bar{z}_{i}\right)\right) \geq \widetilde{v}\left(\sigma, v_{0}\right) s^{n},
$$

for all $s \leq\left(\sqrt{Q_{i}} \sigma\right) / 10,-M_{0} Q^{-1} \leq t \leq 0$ as this is a scale invariant quantity. We have $Q_{i} \rightarrow \infty$ as $i \rightarrow \infty$ since $Q_{i}:=\left|\operatorname{Riem}\left(\bar{z}_{i}, \bar{t}_{i}\right)\right| \geq N_{i}^{2}$, as one sees from (2-2). Let us denote these rescaled solutions also by $\left(M_{i}, g_{i}(t)\right)$. Hence the bound from below for the curvature operator goes to zero as $i \rightarrow \infty$. Taking the pointed limit of a subsequence as $i \rightarrow \infty$ (see Hamilton [8, Theorem 1.2]) of $\left(M_{i}, g_{i}(t), \bar{z}_{i}\right)_{t \in\left(-M_{0}, 0\right]}$, we see that the limiting solution, denoted by $\left(\Omega, p_{0}, h(t)\right)_{t \in\left(-M_{0}, 0\right]}$, has nonnegative curvature operator, is complete, has bounded curvature $|\operatorname{Riem}(x, t)| \leq 4$ at all times and points in the limiting manifold, has $\left|\operatorname{Riem}\left(p_{0}, 0\right)\right|=1$ and $\lim _{r \rightarrow \infty} \operatorname{vol}\left({ }^{t} B_{r}\left(p_{0}\right)\right) / r^{n} \geq \tilde{v}>0$ (note $\tilde{v}=\tilde{v}\left(\sigma, v_{0}, n\right)>0$ does not depend on $\left.M_{0}\right)$. We repeat the procedure for larger and larger $M_{0}, M_{0} \rightarrow \infty$ to obtain, after taking a pointed limit of a subsequence, a solution $\left(\tilde{\Omega}, \tilde{p}_{0}, \tilde{h}(t)\right)_{t \in(-\infty, 0]}$, which has nonnegative curvature operator, is complete, has bounded curvature $|\operatorname{Riem}(x, t)| \leq 4$ at all times and points in the limiting manifold, has $\left|\operatorname{Riem}\left(\tilde{p}_{0}, 0\right)\right|=1$ and $\lim _{r \rightarrow \infty} \operatorname{vol}\left({ }^{t} B_{r}\left(\tilde{p}_{0}\right)\right) / r^{n} \geq \tilde{v}>0$. This is a contradiction to [12, Proposition 11.4]. 
Examining the proof, we see that a bound from below on $\mathcal{R}$ is sufficient to obtain an estimate.

Theorem 2.2 Let $V, r, v_{0}>0,1>\sigma>0$ and $\left(M^{n}, g(t)\right)_{t \in[0, T)}$ be a smooth complete solution to Ricci-flow which satisfies

(a) $\operatorname{vol}\left({ }^{t} B_{r}\left(x_{0}\right)\right) \geq v_{0} r^{n}$,

(b) $\mathcal{R}(x, t) \geq-\frac{V}{r^{2}}$ for all $t \in[0, T), x \in{ }^{t} B_{r}\left(x_{0}\right)$.

Then, there exists a $N=N\left(n, V, v_{0}, \sigma\right)<\infty$ such that

(c) $\mid$ Riem $\mid \leq \frac{N^{2}}{t}+\frac{N^{2}}{r^{2}}$ for all $x \in{ }^{t} B_{r(1-\sigma)}\left(x_{0}\right), t \in\left[0, \frac{1}{r^{2} N^{2}}\right) \cap[0, T)$.

Proof In the use of the Bishop-Gromov estimate in the proof above, we obtain a different constant. Also, the bound from below on $\mathcal{R}$ is now $\mathcal{R} \geq-V$. Otherwise the proof remains unchanged.

\section{A cutoff function and its properties}

In the next section we use a cut off function with certain nice properties. We define this cutoff function here and examine some of its properties.

Lemma 3.1 There exists a smooth cut off function $\varphi:[0, \infty) \rightarrow \mathbb{R}_{0}^{+}$with the following properties:

(i) $0 \leq \varphi \leq 1$;

(ii) $\varphi(r)=1$ for all $r \leq 1, \varphi(r)=0$ for all $r \geq 2$;

(iii) $\varphi$ is decreasing: $\varphi^{\prime} \leq 0$;

(iv) $\varphi^{\prime \prime} \geq-200 \varphi$;

(v) $\left|\varphi^{\prime}\right|^{2} \leq 200 \varphi^{3 / 2}$ for some constant $0<C<\infty$.

Proof To construct a cutoff function with the properties (i)-(iv) stated above is standard. In fact we obtain $\varphi^{\prime \prime} \geq-10 \varphi$ and $\left(\varphi^{\prime}\right)^{2} \leq 10 \varphi$ in place of (iv). Define $\psi=\varphi^{4}$. Then $\psi$ satisfies properties (i)-(iii) trivially, and $\psi^{\prime}=\left(\varphi^{4}\right)^{\prime}=4 \varphi^{3} \varphi^{\prime}$. Then $\left(\psi^{\prime}\right)^{2} \leq 16 \varphi^{6}\left(\varphi^{\prime}\right)^{2} \leq 160 \varphi^{7}=160\left(\varphi^{4}\right)^{7 / 4}=160 \psi^{7 / 4} \leq 160 \psi^{6 / 4}=160 \psi^{3 / 2}$ since $\varphi \leq 1$. Also $\psi^{\prime \prime}=\left(\varphi^{4}\right)^{\prime \prime}=\left(4 \varphi^{3} \varphi^{\prime}\right)^{\prime}=12 \varphi^{2}\left|\varphi^{\prime}\right|^{2}+4 \varphi^{3} \varphi^{\prime \prime} \geq-40\left(\varphi^{3}\right) \varphi=-40 \psi$. Hence (iv) and (v) are also satisfied. 
Lemma 3.2 Let $A, B>0$. We may choose a cutoff function satisfying

(i) $0 \leq \varphi \leq 1$,

(ii) $\varphi(r)=1$ for all $r \leq A, \varphi(r)=0$ for all $r \geq A+B$,

(iii) $\varphi$ is decreasing: $\varphi^{\prime} \leq 0$,

(iv) $\varphi^{\prime \prime} \geq-k_{0}(A, B) \varphi,\left(\varphi^{\prime}\right)^{2} \leq k_{0}(A, B) \varphi$,

(v) $\left|\varphi^{\prime}\right|^{2} \leq k_{0}(A, B) \varphi^{3 / 2}$ for some constant $0<k_{0}(A, B)<\infty$.

Proof By shifting and scaling: Define $\widetilde{\varphi}(r):=\varphi\left(\frac{r+B-A}{B}\right)$ where $\varphi$ is the function appearing Lemma 3.1. Then $\tilde{\varphi}$ has all of the desired properties.

\section{Construction of a cutoff function on a Riemannian manifold which is evolving by Ricci flow}

Now we construct a cutoff function similar to that constructed by G Perelman (see proof of [12, Theorem 10.1]) and similar to that used by B-L Chen in [4]. Assume that we have a solution to Ricci flow $(M, g(t))_{t \in[0, T)}$. We do not assume that the curvature is bounded uniformly on some region for all $t \in[0, T)$ as in the argument of B-L Chen in the proof of [4, Proposition 2.1]. Instead we assume a uniform estimate of the form

(c) $\mid$ Riem $\mid \leq \frac{c_{0}}{t}$ on ${ }^{t} B_{1 / 4}\left(x_{0}\right)$ for $t \in[0, S)$,

for some $S \leq \frac{1}{100}, S \leq T$. Note: The radius of the ball $1 / 4$ is chosen for convenience. If we replace $1 / 4$ by $\sigma>0$, then all constants occurring in this section also depend on $\sigma$. This estimate combined with [12, Lemma 8.3] guarantees that the cutoff function we construct will satisfy estimates which are sufficient for the arguments in the following section.

Let $\varphi:[0, \infty) \rightarrow \mathbb{R}_{0}^{+}$be one of the cutoff functions defined above with $A \leq 1$.

Let $r_{0}(t)=\sqrt{t}, K(t)=\frac{c_{0}}{t}$. Then $|\operatorname{Ric}(x, t)| \leq(n-1) K$ whenever $d\left(x, x_{0}, t\right) \leq r_{0}(t)$ for all $t \leq S$ in view of (c). Hence, using [12, Lemma 8.3], we have

$$
\begin{aligned}
\frac{\partial}{\partial t} d_{t}(x)-\Delta d_{t}(x) & \geq-(n-1)\left(\frac{2}{3} K r_{0}+r_{0}^{-1}\right) \\
& =-(n-1) \frac{\left((2 / 3) c_{0}+1\right)}{\sqrt{t}},
\end{aligned}
$$

by condition (c), where this inequality is valid for points $(x, t)$ where $d_{t}(x)=d\left(x_{0}, x, t\right)$ is differentiable and $t \leq S$, and $d\left(x_{0}, x, t\right) \geq r_{0}(t)=\sqrt{t}$. Note for $t \leq A^{2} / 10$, the last condition is satisfied for all $x$ outside of ${ }^{t} B_{A / 2}\left(x_{0}\right)$. The right-hand side is integrable. 
That is,

$$
\begin{aligned}
\frac{\partial}{\partial t}\left(d_{t}(x)+4(n-1)\left(c_{0}+1\right) \sqrt{t}\right)-\Delta\left(d_{t}(x)+4(n-1)\left(c_{0}+1\right) \sqrt{t}\right) & \\
& \geq \frac{2(n-1)\left(c_{0}+1\right)}{\sqrt{t}}>0
\end{aligned}
$$

for such points. Denote the constant appearing here as $m_{0}=m_{0}\left(c_{0}, n\right)=(n-1)\left(c_{0}+1\right)$. Let $k(x, t)=\varphi\left(d_{t}(x)+4 m_{0} \sqrt{t}\right)$. Using the above information, we obtain the following evolution inequality for $k$ :

$$
\begin{aligned}
\left(\frac{\partial}{\partial t}-\Delta\right) k & =\varphi^{\prime} \cdot\left(\frac{\partial}{\partial t}-\Delta\right)\left(d_{t}+4 m_{0} \sqrt{t}\right)(x, t)-\left(\varphi^{\prime \prime}\right) \\
& \leq \varphi^{\prime} \frac{m_{0}}{2 \sqrt{t}}+k_{0} \varphi \leq k_{0} \varphi,
\end{aligned}
$$

where $k_{0}=k_{0}(A, B)$ comes from Lemma 3.2. Note that $\varphi\left(d_{t}(x)+4 m_{0} \sqrt{t}\right)=1$ for all $x \in{ }^{t} B_{A / 2}\left(x_{0}\right)$ and $t \leq A^{2} /\left(100 m_{0}^{2}\right)$ and hence $\varphi^{\prime}=0$ for all points $x$ inside ${ }^{t} B_{A / 2}\left(x_{0}\right)$ as long as $t \leq A^{2} /\left(100 m_{0}^{2}\right)$, and hence the above evolution inequality (3-1) is valid for all $x \in M$ and all $t \leq A^{2} /\left(100 m_{0}^{2}\right)$ (we assume that $\left.m_{0} \gg 1\right)$ as long $(x, t)$ is a point where $d_{t}(x)=d\left(x_{0}, x, t\right)$ is differentiable. Hence $h(x, t):=e^{-2 k_{0} t} k(x, t)$ satisfies

$$
\left(\frac{\partial}{\partial t}-\Delta\right) h(x, t) \leq 0
$$

for all $x$ and all $t \leq A^{2} /\left(100 m_{0}^{2}\right)$, as long as $d\left(x_{0}, \cdot, \cdot\right)$ is differentiable there. We collect the definitions and observations made above in the following.

Proposition 3.3 Let $(M, g(t))_{t \in[0, T)}$ by a smooth complete solution to Ricci flow and $\varphi$ be one of the functions appearing in Lemma 3.2. with $A \leq 100$. We assume that

(c) $\mid$ Riem $\mid \leq \frac{c_{0}}{t}$ on ${ }^{t} B_{1 / 4}\left(x_{0}\right)$ for $t \in[0, S)$,

for some $S \leq 1 / 100$ and $S \leq T$. Then we have that $h: M \rightarrow \mathbb{R}$ is the function $h(x, t):=e^{-2 k_{0} t}\left(\varphi\left(d_{t}(x)+4 m_{0} \sqrt{t}\right)\right)$ where $d_{t}(x):=d_{t}\left(x_{0}, x\right)$, and $x_{0}$ is a fixed point in $M$ and $m_{0}=m_{0}\left(c_{0}, n\right)=(n-1)\left(c_{0}+1\right), k_{0}=k_{0}(A, B)$. For $t \leq A^{2} /\left(100 m_{0}^{2}\right)$, we have

$$
\left(\frac{\partial}{\partial t}-\Delta\right) h(x, t) \leq 0
$$

as long as $d\left(x_{0}, \cdot, \cdot\right)$ is differential at $(x, t) . h \equiv 0$ for all $d_{t}(x) \geq(A+B)$ and $h \equiv e^{-2 k_{0} t}$ for all $d_{t}(x) \leq A-4 m_{0} \sqrt{t}$ and $h(x, t) \leq e^{-2 k_{0} t} \leq 1$. 


\section{A local result in two dimensions}

In this section we restrict ourselves to the two-dimensional case. We consider a ball of radius $r$ in a two-dimensional manifold which has curvature operator and volume bounded from below by known constants. We show that a ball of smaller radius will smooth out quickly at least for a short time. The rate of this smoothing depends on the bounds from below and $r$.

Theorem 4.1 Let $\left(M^{2}, g(t)\right)_{t \in[0, T)}$ be a smooth complete solution to Ricci flow and let $x_{0} \in M, N, v_{0}, r>0$ and $1>\sigma, \alpha>0$. Assume that

- $\quad \operatorname{vol}{ }^{0} B_{r}\left(x_{0}\right) \geq v_{0} r^{2}$,

- $\mathrm{R}(g(0)) \geq-\frac{N}{r^{2}}$ on ${ }^{0} B_{r}\left(x_{0}\right)$,

and $1>\sigma>0$. Then there exists a $\widetilde{v}_{0}=\widetilde{v}_{0}\left(v_{0}, \sigma, N, \alpha\right)>0$ and a $\delta_{0}=\delta_{0}\left(v_{0}, \sigma, N, \alpha\right)>0$ such that

- $\operatorname{vol}\left({ }^{t} B_{r}\left(x_{0}\right)\right) \geq \widetilde{v}_{0} r^{2}$,

- $\mathrm{R}(g(t)) \geq-\frac{(N+\alpha)}{r^{2}}$ on ${ }^{t} B_{(1-\sigma) r}\left(x_{0}\right)$,

- $|\mathrm{R}(g(t))| \leq \frac{1}{\delta_{0} t}$ on ${ }^{t} B_{(1-\sigma) r}\left(x_{0}\right)$,

as long as $t \leq r^{2}\left(\delta_{0}\right)^{2}$ and $t \in[0, T)$.

Remark 4.2 In [4, Theorem 3.1] B-L Chen proved the following similar result: if we assume the above conditions with $r=1$ but replace the lower bound on the scalar curvature by the condition that $\left|\mathrm{R}\left(g_{0}\right)\right| \leq 1$ on ${ }^{0} B_{2}\left(x_{0}\right)$, then $|\mathrm{R}(g(t))| \leq 2$ for all $t \in\left[0, T\left(n, v_{0}\right)\right)$ on a smaller ball. This a version of $\mathrm{G}$ Perelman's second pseudolocality result, [12, Theorem 10.3], in dimension two. Note that in this case, the curvature bound and volume bound from below guarantee that balls of radius $r \leq R=R\left(n, \epsilon, v_{0}\right)$ which are sufficiently small satisfy the almost Euclidean condition $\operatorname{vol}\left({ }^{0} B_{r}\left(x_{0}\right)\right) \geq(1-\epsilon) r^{2}$.

Proof By scaling, it suffices to prove the theorem for $r=1$. Without loss of generality, $\sigma>1 / 2$. By the Bishop-Gromov volume comparison principle, we have that $\operatorname{vol}\left({ }^{0} B_{s}\left(x_{0}\right)\right) \geq c\left(N, v_{0}\right) s^{n}$ for all $s \leq 1$. In particular, we have that $\operatorname{vol}\left({ }^{0} B_{1 / 1000}\left(x_{0}\right)\right) \geq \widetilde{v}_{0}\left(N, v_{0}\right)>0$. For some maximal time interval $\left[0, S_{\max }\right)$, $0<S_{\max } \leq T$ we have (due to smoothness) that 
(a) $\mathrm{R} \geq-(N+2 \alpha)$ on ${ }^{t} B_{(1-\sigma)}\left(x_{0}\right)$,

(b) $\operatorname{vol}\left({ }^{t} B_{1 / 1000}\left(x_{0}\right)\right) \geq \frac{\widetilde{v}_{0}}{2}$.

Note that (b) implies $(\tilde{\mathrm{b}}): \operatorname{vol}\left({ }^{t} B_{1}\left(x_{0}\right)\right) \geq \frac{\widetilde{v}_{0}\left(N, v_{0}\right)}{2}>0$ trivially.

Our aim is to obtain an estimate from below for the time $S_{\max }$ which only depends on $N, v_{0}, \sigma, \alpha$ ( $n=2$ is fixed here). According to Theorem 2.1 above, we have that

(c) $|\mathrm{R}| \leq \frac{c_{0}\left(\widetilde{v}_{0}, N, \sigma, \alpha\right)}{t}$ on ${ }^{t} B_{1 / 4}\left(x_{0}\right)$

$$
\text { for } t \in\left[0, S_{\max }\right) \cap\left[0, S\left(N, \widetilde{v}_{0}, \sigma, \alpha\right)\right)=:\left[0, S\left(N, v_{0}, \sigma, \alpha\right)\right) \text {, }
$$

for some constant $c_{0}=c_{0}\left(N, \widetilde{v}_{0}, \sigma, \alpha\right)=c_{0}\left(N, v_{0}, \sigma, \alpha\right)$. In the rest of the proof we often shorten time intervals $\left[0, S\left(N, v_{0}, \sigma, \alpha\right)\right)$ to $\left[0, \widetilde{S}\left(N, v_{0}, \sigma, \alpha\right)\right)$, where we have $0<\tilde{S}\left(N, v_{0}, \sigma, \alpha\right)<S\left(N, v_{0}, \sigma, \alpha\right)$. We will denote $\widetilde{S}\left(N, v_{0}, \sigma, \alpha\right)$ also by $S\left(N, v_{0}, \sigma, \alpha\right)$.

Claim (i) The scalar curvature is bounded from below by $-(N+\alpha)$ on ${ }^{t} B_{(1-\sigma)}\left(x_{0}\right)$ for $t \leq S$ where $S=S\left(v_{0}, \sigma, \alpha, N\right)>0$, as long as $t \leq S_{\max }$. That is, (a) is not violated for $t \leq S$ as long as (b) still holds.

\section{Proof of Claim (i)}

We modify the argument of B-L Chen given in the proof of [4, Theorem 3.6]. Let $f:=h \mathrm{R}$ where we have chosen $\varphi$ in the definition of $h$ of Proposition 3.3 to be a smooth function with $\varphi(r)=0$ for $r \geq\left(1-\frac{\sigma}{4}\right)$, and $\varphi(r)=1$ for $r \leq\left(1-\frac{\sigma}{2}\right)$. Note that $m_{0}=m_{0}\left(c_{0}, n\right)=m_{0}\left(c_{0}\left(v_{0}, N, \sigma, \alpha\right), 2\right)=m_{0}\left(v_{0}, \sigma, \alpha, N\right)$ in this case, where $m_{0}$ is the constant appearing in Proposition 3.3. In the following we assume without loss of generality, that $t \leq\left(A^{2}=\left(1-\frac{\sigma}{2}\right)^{2}\right) /\left(100 m_{0}^{2}\right)$, so that Proposition 3.3 is valid.

Using the evolution inequality for $h$ and the evolution equation for $\mathrm{R}$ we see that at any point $(x, t)$ where $\mathrm{R}(x, t)<0$ and $d\left(x_{0}, \cdot, \cdot\right): M \times[0, T) \rightarrow \mathbb{R}$ is differentiable we have

$$
\begin{aligned}
\frac{\partial}{\partial t}(h \mathrm{R}+\sqrt{t})- & \Delta(h \mathrm{R}+\sqrt{t}) \\
= & \mathrm{R}\left(\frac{\partial}{\partial t}-\Delta\right)(h)+h\left(\frac{\partial}{\partial t}-\Delta\right)(\mathrm{R})-2 g(\nabla h, \nabla \mathrm{R})+\frac{1}{2 \sqrt{t}} \\
\geq & 2 h|\mathrm{Ric}|^{2}-2 g(\nabla h, \nabla \mathrm{R})+\frac{1}{2 \sqrt{t}} .
\end{aligned}
$$


If $(x, t)$ is a first time and point where $(h(x, t) \mathrm{R}(x, t)+\sqrt{t})=-\left(N+\frac{\alpha}{2}\right)$ then the gradient term at $(x, t)$ can be estimated as follows:

$$
\begin{aligned}
-2 g(\nabla \mathrm{R}, \nabla h) & =-\frac{2}{h} g(\nabla(\mathrm{R} h), \nabla h)+2 \mathrm{R} \frac{|\nabla h|^{2}}{h} \\
& =2 \mathrm{R} \frac{|\nabla h|^{2}}{h} \\
& \geq-\frac{(\mathrm{R})^{2}}{4} h-\left(\frac{4}{h^{3}}\right)|\nabla h|^{4} \\
& \geq-\frac{(\mathrm{R})^{2}}{4} h-2 C(\sigma),
\end{aligned}
$$

where in the last line we have used that $|\nabla d|=1$ and $\left|\varphi^{\prime}\right|^{2} \leq C(A, B) \varphi^{3 / 2}$ with $B=\frac{\sigma}{4}, A=\left(1-\frac{\sigma}{2}\right)$. Now using this inequality in (4-1) we get

$$
\frac{\partial}{\partial t}(h \mathrm{R}+\sqrt{t})-\Delta(h \mathrm{R}+\sqrt{t}) \geq 2 h|\mathrm{Ric}|^{2}-\frac{(\mathrm{R})^{2}}{n} h-2 C+\frac{1}{2 \sqrt{t}}>0
$$

at the point $(x, t)$ in question, since $(h(x, t) \mathrm{R}(x, t)+\sqrt{t})=-\left(N+\frac{\alpha}{2}\right)$ guarantees that $\mathrm{R}(x, t)<0$, as long as $d\left(x_{0}, \cdot, \cdot\right)$ is differentiable at $(x, t)$ and $t \leq S\left(v_{0}, N, \sigma, \alpha\right)$. Hence, in view of the maximum principle, we see $h \mathrm{R}+\sqrt{t} \geq 0$ for all $t \leq S\left(N, \sigma, v_{0}, \alpha\right)$ as long as $t \leq S_{\max }$ (for the case that $(x, t)$ is not a point where $d$ is differentiable, then the argument is still valid, as we explain in Claim (iii) at the end of the proof). In particular, this shows $\mathrm{R} \geq-\left(N+\frac{3 \alpha}{4}\right)$ for $x \in{ }^{t} B_{1-\sigma}\left(x_{0}\right)$ as long as $t \leq S\left(N, \sigma, v_{0}, \alpha\right)$ (possibly a smaller $S$ ) and $t \leq S_{\max }$, in view of the definition $h(x, t):=e^{-2 k_{0} t}\left(\varphi\left(d_{t}(x)+8 m_{0} \sqrt{t}\right)\right.$, which is close as we like to one on ${ }^{t} B_{1-\sigma}\left(x_{0}\right)$ for $t \leq S\left(N, \sigma, v_{0}, \alpha\right)$ small enough.

This finishes the proof of the Claim (i).

Claim (ii) The volume condition (b) is not violated for a well-defined time interval, if (a) holds. Let $x, y \in{ }^{t} B_{1 / 100}\left(x_{0}\right)$. Then $d(x, y, t) \leq d\left(x, x_{0}, t\right)+d\left(y, x_{0}, t\right) \leq \frac{2}{100}$ and hence any shortest geodesic between $x$ and $y$ must lie in ${ }^{t} B_{1 / 4}\left(x_{0}\right)$ (proof: if it did not, smooth out the union of the two radial curves (measured with respect to $g(t)$ ) going from $x$ to $x_{0}$ and then back to $y$ of length $2 / 100$. This would result in a curve of length less then $3 / 100$. Any curve which starts in ${ }^{t} B_{1 / 100}\left(x_{0}\right)$, reaches $\partial^{t} B_{1 / 4}\left(x_{0}\right)$ and finishes in ${ }^{t} B_{1 / 100}\left(x_{0}\right)$ must have length larger than or equal to $1 / 20$. Hence, if a minimising Geodesic between $x$ and $y$ leaves ${ }^{t} B_{1 / 4}\left(x_{0}\right)$ we obtain a contradiction). Hence using the estimate of Hamilton (see [9, Theorem 17.2] and the editors' comment thereon in [2] or, alternatively, see [15, Appendix B]) and the fact that (a) and (c) hold 
on ${ }^{t} B_{1 / 4}\left(x_{0}\right)$, we get

$$
\begin{aligned}
& -(N+2 \alpha) d(y, x, t) \geq \frac{\partial}{\partial t} d(y, x, t) \geq-\frac{c_{1}\left(c_{0}\right)}{\sqrt{t}}, \\
& \qquad \text { for all } s \leq t \leq \min \left(S_{\max }, S\left(N, v_{0}, \sigma, \alpha\right)\right), \quad x, y \in{ }^{t} B_{1 / 100}\left(x_{0}\right),
\end{aligned}
$$

where $r \geq(\partial / \partial t) d \geq m$ is meant in the sense of forward difference quotients (see Hamilton [9, Theorem 17.2]). Note $c_{1}\left(c_{0}\right)=c_{1}\left(v_{0}, \sigma, N, \alpha\right)$. Integrating in time we get

$e^{-(N+\alpha)(t-s)} d\left(x_{0}, x, s\right) \geq d(x, y, t) \geq d(x, y, s)-2 c_{1} \sqrt{t}$,

for all $s \leq t \leq \min \left(S_{\max }, S\left(N, v_{0}, \sigma, \alpha\right)\right), \quad x, y \in{ }^{t} B_{1 / 100}\left(x_{0}\right)$.

Arguing as in [15, Corollary 6.2], we see that $\operatorname{vol}\left({ }^{t} B_{1 / 1000}\left(x_{0}\right)\right) \geq(3 / 4) \widetilde{v}_{0}$ for all $t \leq S\left(N, v_{0}, \sigma, \alpha\right)$ (we have possibly decreased $S$ once again here) as long as $t \leq S_{\max }$. That is the volume condition is not violated for the time interval $\left[0, S\left(N, \sigma, v_{0}, \alpha\right)\right]$ (as long as $t \leq S_{\max }$ ).

In particular we see that the second condition (b) will not be violated for some well defined time interval $\left[0, S\left(N, \sigma, v_{0}, \alpha\right)\right]$ (as long as $t \leq S_{\max }$ ). This finishes the proof of Claim (ii) and of the theorem if we accept Claim (iii) below.

Claim (iii) If $d_{t}(\cdot)$ is not differentiable at $x \in M$ then we use the trick of E Calabi [1] as follows. Let $y_{0}$ be a point on a shortest geodesic between $x_{0}$ and $x$ which is very close to $x_{0}$. By smoothness, we can find a small open neighbourhood $P$ of $(x, t)$ in $M \times(0, T)$ such that $d\left(y_{0}, \cdot, s\right)$ is differentiable at $y$ for each $(y, s) \in P$. We define $\tilde{d}_{s}(y)=d\left(x_{0}, y_{0}, s\right)+d\left(y_{0}, y, s\right)$. Then $\widetilde{d}_{s}(\cdot)$ is differentiable at $y$ for all $(y, s) \in P$. Furthermore, $\tilde{d}_{s}(\cdot) \geq d_{s}(\cdot)$ for all $(y, s) \in P$ due to the triangle inequality. Since $\varphi$ is nonincreasing, we therefore have $\widetilde{k}=\varphi\left(\tilde{d}+m_{0} \sqrt{s}\right) \leq \varphi\left(d+m_{0} \sqrt{s}\right)=k$ in $P$ and per definition $\widetilde{k}(x, t)=k(x, t)$ if $(x, t)$ is the point given at the beginning of the claim. Also, if we pick $y_{0}$ very close to $x_{0}$ we still have

$$
\frac{\partial}{\partial t}\left(\tilde{d}_{t}(x)+m_{0} \sqrt{t}\right)-\Delta\left(\tilde{d}_{t}(x)+m_{0} \sqrt{t}\right) \geq \frac{m_{0}}{2 \sqrt{t}}>0,
$$

where here $m_{0}$ is the constant appearing in Proposition 3.3. Hence we may argue with $\tilde{h}(y, s):=e^{-2 k_{0} s} \tilde{k}(y, s)$ everywhere above. If for example $(x, t)$ is a first time and point where $(h(x, t) \mathrm{R}(x, t)+\sqrt{t})=-(N+\alpha / 2)$, then $(x, t)$ is a first time and point for which the function $(\tilde{h}(x, t) \mathrm{R}(x, t)+\sqrt{t})=-(N+\alpha / 2)$ on the set $P$ and hence we may argue as above with $(h(x, t) \mathrm{R}(x, t)+\sqrt{t})$ replaced by $(\tilde{h}(x, t) \mathrm{R}(x, t)+\sqrt{t})$ (without loss of generality $\mathrm{R}<0$ on $P$, since $\mathrm{R}(x, t)<0$ and hence $\widetilde{h}(y, s) \mathrm{R}(y, s)+\sqrt{t} \geq h(y, s) \mathrm{R}(y, s)+\sqrt{t} \geq-(N+\alpha / 2)$ on $P \cap\{(y, s) \mid s \leq t\})$. 
We must also consider the case that $\tilde{d}_{(\cdot)}(x)$ is not differentiable in time at the time $t$ we are considering. In this case, all the estimates are still valid if we understand the inequalities $(\partial / \partial t) \tilde{d}_{t}(x) \geq m$ or $(\partial / \partial t) \tilde{d}_{t}(x) \leq m$ in the sense of forward difference quotients; see Hamilton [7]. At times $s<t$ very close to $t((x, t)$ as above), we have (due to smoothness)

$$
\begin{gathered}
\Delta(\tilde{h}(x, s) \mathrm{R}(x, s)+\sqrt{s}) \geq-e, \\
|\nabla(\tilde{h}(x, s) \mathrm{R}(x, s))| \leq e,
\end{gathered}
$$

where $e$ is as small as we like. Remembering that $\tilde{h}(x, t)>0$, we see that the term $-(2 / \tilde{h}(x, s)) g(\nabla(\mathrm{R} \tilde{h}), \nabla \tilde{h})(x, s)$ which is zero at $(x, t)$ is also as small as we like for $s<t$ very close to $t$. Hence, examining the proof of Claim (i) again, we see that

$$
\frac{\partial}{\partial t}(\tilde{h}(x, s) \mathrm{R}(x, s)+\sqrt{s})>0
$$

in the sense of forward difference quotients for $s<t$ close to $t$.

In particular, using [7, Lemma 3.1], we see that $\tilde{h}(x, t) \mathrm{R}(x, t)+\sqrt{t}>-(N+\alpha / 2)$, which is a contradiction. Hence, there is no such $(x, t)$.

\section{A local result in three dimensions}

In this section we restrict ourselves to the three-dimensional case. We first consider a ball of radius 1 in a three-dimensional manifold which has curvature operator and volume bounded from below by known constants at time zero. For later times we assume a bound on the curvature of the form $|\operatorname{Riem}(g(t))| \leq \frac{N}{t}$ on the time $t$ ball of radius 1, where $N$ depends on the curvature bound from below. We show that the curvature can not become too negative too quickly on smaller balls.

Theorem 5.1 Let $v_{0}>0$ and $N \geq 1$ be given. Let $\left(M^{3}, g(t)\right)_{t \in[0, T)}$ be a smooth complete solution to Ricci flow with $T \leq 1$, and let $x_{0} \in M$ be a point such that

- $\quad \operatorname{vol}^{0} B_{1}\left(x_{0}\right) \geq v_{0}$,

- $\mathcal{R}(g(0)) \geq-\frac{1}{400 N}$ on ${ }^{0} B_{1}\left(x_{0}\right)$,

- $|\operatorname{Riem}(g(t))|(x) \leq N / t$ for all $t \in(0, T)$ for all $x \in{ }^{t} B_{1}\left(x_{0}\right)$.

Then, for all $1>\sigma>0$ there exist $\delta=\delta\left(v_{0}, N, \sigma\right)>0, \widetilde{v}_{0}=\widetilde{v}_{0}\left(N, v_{0}, \sigma\right)>0$ so that

- $\operatorname{vol}\left({ }^{t} B_{1}\left(x_{0}\right)\right) \geq \widetilde{v}_{0}$,

- $\mathcal{R}(g(t)) \geq-1$ on ${ }^{t} B_{(1-\sigma)}\left(x_{0}\right)$,

as long as $t \leq\left(\delta_{0}\right)^{2}$ and $t \in[0, T)$. 
Proof We have $\operatorname{vol}\left({ }^{0} B_{s}\left(x_{0}\right)\right) \geq c\left(N, v_{0}\right) s^{n}$ for all $s \leq 1$, by the Bishop-Gromov volume comparison principle. In particular vol $\left({ }^{0} B_{1 / 1000}\left(x_{0}\right)\right) \geq \widetilde{v}_{0}\left(N, v_{0}\right)>0$.

For some maximal time interval $\left[0, S_{\max }\right), S_{\max } \leq T$ we have (due to smoothness) that

(a) $\mathcal{R} \geq-1$ on ${ }^{t} B_{(1-\sigma)}\left(x_{0}\right)$,

(b) $\operatorname{vol}\left({ }^{t} B_{1 / 1000}\left(x_{0}\right)\right) \geq \frac{\widetilde{v}_{0}}{2}$.

Our aim is to obtain an estimate from below for the time $S$ which only depends on $v_{0}$ and $N$ ( $n=3$ is fixed here).

For convenience we denote the constant $\frac{1}{10 N}$ by $\epsilon_{0}:=\frac{1}{10 N}$.

Claim (i) The scalar curvature is bounded from below by $-2 \epsilon_{0}$ on ${ }^{t} B_{(1-\sigma / 10)}\left(x_{0}\right)$ for $t \leq S$ where $S=S\left(v_{0}, \sigma, N\right)>0$, as long as $t \leq S_{\max }$.

Proof of Claim (i) This is proved using the same argument as that given in the proof of Claim (i) in the proof of Theorem 4.1 above.

This finishes the proof of the Claim (i).

For convenience we introduce $\alpha<\beta<\gamma$ to be the eigenvalues of $\mathcal{R}$ as in Hamilton. Then $\mathrm{R}=\alpha+\beta+\gamma$ and $|\mathrm{Ric}|^{2}=1 / 2\left(\alpha^{2}+\beta^{2}+\gamma^{2}+\alpha \beta+\alpha \gamma+\beta \gamma\right)$.

Claim (ii) $\alpha+2 \mathrm{R} \geq-2 \epsilon_{0}$ on ${ }^{t} B_{(1-\sigma / 5)}\left(x_{0}\right)$ as long as $t \leq S_{\max }$ and $t \leq S\left(N, \sigma, v_{0}\right)$.

Remark 5.2 A local result of this type was first shown by B-L Chen under the extra assumption that the Ricci curvature remains bounded on ${ }^{t} B_{1}\left(x_{0}\right)$; see [4, Proposition 2.2] (for a related result see [14, Lemma 4.1]).

Proof of Claim (ii) Let $L(V, V)=\operatorname{Riem}(V, V)+2 \mathrm{R} \operatorname{Id}(V, V), P(V, V)=h L(V, V)+$ $\epsilon_{0}\left(1+k t / \epsilon_{0}\right)(=h(\operatorname{Riem}(V, V)+2 \mathrm{R} \operatorname{Id}(V, V))+\epsilon)$ for 2 -forms, where we have chosen now $\varphi$ to satisfy $\varphi(r)=1$ for all $r \leq(1-\sigma / 6)$ and $\varphi(r)=0$ for all $r \geq(1-\sigma / 7)$ in the definition of $h$ in Proposition 3.3. We shall only be concerned with points where $h \neq 0$, and so we may use freely the results of Claim (i) for $t \leq S\left(N, v_{0}, \sigma\right)$. We do so, sometimes without further comment. $k=k\left(N, \sigma, v_{0}\right)$ is a large constant which we shall choose later in the proof.

Also we have introduced the notation $\epsilon=\epsilon(t)=\epsilon_{0}\left(1+k t / \epsilon_{0}\right)$. For the time intervals we are considering, we have $\epsilon_{0} \leq \epsilon(t) \leq 2 \epsilon_{0}$, as we shall assume that $t \leq \epsilon_{0} /(2 k)$.

In all of the following arguments (also for the proofs of Claims (iii), (iv) and (v)) we shall be calculating the evolution of the curvature in the setting of [7]. That is, we are using 
the trick of K Uhlenbeck. In particular, the metric $G_{a b}(x):=g_{i j}(x, t) u_{a}^{i}(x, t) u_{b}^{j}(x, t)$ is the pullback of the metric $g(x, t)$, and it is time independent: $(\partial / \partial t) G_{a b}(x)=0$. Id here is the operator on two forms given by $\operatorname{Id}(V, W):=G^{a b} G^{c d} V_{a c} W_{b d}$. In particular $(\partial / \partial t)(\operatorname{Id}(V, W))=0$ for a time independent vector field. The connection, ${ }^{t} \nabla$, is the pullback connection of ${ }^{g(t)} \nabla$. We still have ${ }^{t} \Delta f(x)=\Delta_{g(t)} f(x)$ for smooth functions $f: M \rightarrow \mathbb{R}$ (the left hand side is the Laplacian with respect to the pullback connection and the right hand side is the Laplacian with respect to $g(t))$. We also have ${ }^{t} \nabla \mathrm{Id}=0$; see [7] for details. Once again we consider only $t \leq A^{2} /\left(100 m_{0}^{2}\right)=\tilde{m}_{0}\left(\sigma, N, v_{0}\right)$ so that Proposition 3.3 is applicable. Then $P(V, V)=h(\alpha+2 \mathrm{R})+\epsilon$ for a 2 -form $V$ with length one which minimises $P$ at any point in space and time. We first estimate the reaction term coming from the evolution equation for $L$. At the end of the proof we explain how to deal with the reaction diffusion equation for $P$ (in particular the gradient terms). In [14, Lemma 4.1] it is shown (with $\epsilon:=1$ there) that the reaction equation for $L=\alpha+2 R$ is given by

$$
\frac{\partial}{\partial t}(\alpha+2 \mathrm{R})=\alpha^{2}+\beta \gamma+2\left(\alpha^{2}+\beta^{2}+\gamma^{2}+\alpha \beta+\alpha \gamma+\beta \gamma\right)
$$

In case $\beta, \gamma \geq 0$, or $\beta, \gamma \leq 0$ (which implies $\beta \gamma \geq 0$ ) we get

$$
\begin{aligned}
\frac{\partial}{\partial t}(\alpha+2 \mathrm{R}) & \geq \alpha^{2}+2\left(\alpha^{2}+\beta^{2}+\gamma^{2}+\alpha \beta+\alpha \gamma\right) \\
& =3 \alpha^{2}+2 \beta^{2}+2 \gamma^{2}+2 \alpha \beta+2 \alpha \gamma \\
& \geq\left(\alpha^{2}+\gamma^{2}+\beta^{2}\right) \\
& \geq \frac{1}{1000}(\alpha+2 \mathrm{R})^{2},
\end{aligned}
$$

in view of Young's Inequality. In case $\beta \leq 0, \gamma \geq 0$ (which implies that $\alpha \beta \geq 0$ ) we get by applying Young's Inequality a number of times

$$
\begin{aligned}
\frac{\partial}{\partial t}(\alpha+2 \mathrm{R}) & =\alpha^{2}+\beta \gamma+2\left(\alpha^{2}+\beta^{2}+\gamma^{2}+\alpha \beta+\alpha \gamma+\beta \gamma\right) \\
& \left.\geq 3 \alpha^{2}+2 \beta \gamma+2 \alpha \gamma+2 \beta^{2}+2 \gamma^{2}\right) \\
& \geq 2 \alpha^{2}+2 \beta \gamma+2 \beta^{2}+\gamma^{2} \\
& \geq \frac{1}{3}\left(\alpha^{2}+\beta^{2}+\gamma^{2}\right) \\
& \geq \frac{1}{1000}(\alpha+2 \mathrm{R})^{2} .
\end{aligned}
$$

At a first time and point $(y, s)$ where $h(\alpha+2 \mathrm{R})=-\epsilon$, we must clearly have that $\alpha<0$ (otherwise $-\epsilon=h(\alpha+2 \mathrm{R}) \geq 0$ which is a contradiction). Let $V$ be a 2 -form 
with length one such that $P(V, V)=0$. We have

$$
\begin{aligned}
\frac{\partial}{\partial t} P(V, V) \geq(\Delta P)(y, s)(V, V)-2\left(g^{i j} \nabla_{j} h\right)(y, s)\left(\nabla_{i} L\right)(y, s)(V, V) \\
+\frac{1}{1000} h(y, s)(L(y, s)(V, V))^{2}+k
\end{aligned}
$$

in view of the above reaction equation for $L$.

We estimate the gradient term in the above as follows:

$$
\begin{aligned}
-2 g^{i j} & \nabla_{j} h(y, s)\left(\nabla_{i} L\right)(y, s)(V, V) \\
& =-\frac{2}{h(y, s)}\left(g^{i j} \nabla_{j} h\right)(y, s)\left(\nabla_{i}(h L)\right)(y, s)(V, V) \\
& =2 L(y, s)(V, V)\left(\frac{|\nabla h|^{2}}{h}\right)(y, s) \quad+2 L(y, s)(V, V) \frac{|\nabla h|^{2}}{h}(y, s) \\
& \geq-\frac{L^{2}}{2000}(y, s) h(y, s)-\frac{4000}{h^{3}(y, s)}|\nabla h|^{4}(y, s) \\
& \geq-\frac{L^{2}}{2000}(y, s) h(y, s)-2 \widehat{C},
\end{aligned}
$$

where in the last line we have once again used that $|\nabla h|^{4} \leq \widehat{C}(\sigma) h^{3}$. Hence we obtain

$$
\frac{\partial}{\partial t} P(V, V) \geq(\Delta P)(y, s)(V, V)-2 \widehat{C}+k
$$

at $(y, s)$, which leads to a contradiction if $k$ is chosen appropriately (here $n=3$ ). Hence $P$ remains nonnegative in the time interval considered. In particular, using the definition of $h$, we see that $h(\alpha+2 R)+\epsilon_{0}\left(1+k t / \epsilon_{0}\right) \geq 0$ implies

$$
\alpha+2 R+2 \epsilon_{0} \geq 0
$$

on ${ }^{t} B_{(1-\sigma / 5)}\left(x_{0}\right)$ for $t \leq S\left(N, v_{0}, \sigma\right)$ (possibly a smaller $S$ now) as required.

This finishes the proof of Claim (ii).

Claim (iii) The volume condition (b) is not violated for a well defined time interval $t \leq S\left(N, v_{0}, \sigma\right)$, as long as $t \leq S_{\max }$.

The proof may be taken from Claim (ii) in the proof of Theorem 4.1 above, with two changes: we use $\mathcal{R} \geq-1$ on in place of $\mathrm{R} \geq-1$ and we use the assumption that $|\operatorname{Riem}(g(t))| \leq N / t$ on ${ }^{t} B_{1}\left(x_{0}\right)$.

This finishes the proof of Claim (iii). 
Claim (iv) The curvature condition (a) will also not be violated for a well defined time interval $\left[0, S\left(N, v_{0}, \sigma\right)\right)$ as long as $t \leq S_{\max }$. The proof of this claim is initially similar to that of Claim (i) and Claim (ii). In order to estimate the gradient term we require some different arguments.

Define $\epsilon(t):=\epsilon_{0}\left(1 / 2+k t^{1 / 4} / \epsilon_{0}\right)$. Let $Y:=h \mathcal{R}+(\epsilon / 100+\epsilon t \mathrm{R})$ Id (note here $\epsilon t=\epsilon(t) t)$, where $h$ is a cutoff function coming from Proposition 3.3 with $\varphi(r)=1$ for all $r \leq 1-\sigma / 2$ and $\varphi(r)=0$ for all $r \geq 1-\sigma / 3$. This is a local version of the tensor appearing in [14, Lemma 5.2]. $k=k\left(N, \sigma, v_{0}\right)$ is a large positive constant which shall be chosen later in the proof. We wish to show that $Y$ remains larger than zero for $t \leq S\left(N, \sigma, v_{0}\right)$ in ${ }^{t} B_{(1-\sigma / 4)}\left(x_{0}\right)$ as longs as $t \leq S_{\max }$. Assuming we have a first time and point $(y, s), y \in{ }^{t} B_{(1-\sigma / 5)}\left(x_{0}\right)$ and a $2-$ form $V$ of length one where we have $Y(y, s)(V, V)=0$, then we must have that $h(y, s)>0$, because otherwise $Y=h \alpha+(\epsilon t \mathrm{R}+\epsilon / 100)=(\epsilon t \mathrm{R}+\epsilon / 100) \geq-4 \epsilon_{0} \epsilon s+\epsilon / 100>0$ for $s \leq S\left(N, v_{0}, \sigma\right)$ small in view of Claim (i), which is a contradiction. Henceforth, we shall only be concerned with points where $h>0$ and so we may freely use the results of both Claims (i) and (ii) for $t \leq S\left(N, v_{0}, \sigma\right)$ in view of the definition of $\varphi$ we have chosen here. We do so, sometimes without further comment. We assume that $t^{1 / 4} \leq \epsilon_{0} /(100 k)$ so that $1 / 2 \epsilon_{0} \leq \epsilon \leq 2 \epsilon_{0}$. First we examine the reaction term occurring in the evolution of the tensor $h \mathcal{R}+(\epsilon / 100+\epsilon t \mathrm{R}) \mathrm{Id}$. Afterwards we explain how to deal with the reaction diffusion equation occurring here, in particular how to estimate the gradient terms and the zero order term which appears at the end of this estimate. For convenience we introduce $\alpha<\beta<\gamma$ to be the eigenvalues of $\mathcal{R}$ as in [9]. Then $\mathrm{R}=\alpha+\beta+\gamma$ and $|\mathrm{Ric}|^{2}=1 / 2\left(\alpha^{2}+\beta^{2}+\gamma^{2}+\alpha \beta+\alpha \gamma+\beta \gamma\right)$. It is shown in [9] that the reaction equation for $\alpha$ is given by $(\partial / \partial t) \alpha=\alpha^{2}+\beta \gamma$. We have an evolution inequality for $h$ given by $(\partial / \partial t) h \leq \Delta h$ for $t \leq k$ and hence the reaction equation for $h$ may be estimated by $(\partial / \partial t) h \leq 0$ for $t \leq S\left(N, v_{0}, \sigma\right)$. This means that the reaction equation for $h \alpha$ at a point in space and time where $\alpha<0$ and $\gamma>0$ may be estimated by

$$
\begin{aligned}
\frac{\partial}{\partial t} \alpha h \geq h \alpha^{2}+h \beta \gamma & \geq h \alpha^{2}+h \alpha \gamma \\
& =h \alpha^{2}+(h \alpha+\epsilon / 100+\epsilon t \mathrm{R}) \gamma-(\epsilon / 100+\epsilon t \mathrm{R}) \gamma
\end{aligned}
$$

If $\gamma \leq 0$ then $0 \geq \gamma, \beta, \alpha \geq-2 \epsilon_{0}$ in view of Claim (i) and hence we have

$$
\begin{aligned}
\frac{\partial}{\partial t} \alpha h \geq h \alpha^{2}+h \beta \gamma & \geq h \alpha^{2} \\
& \geq h \alpha^{2}+h \alpha \gamma-10 \\
& =h \alpha^{2}+(h \alpha+\epsilon / 100+\epsilon t \mathrm{R}) \gamma-(\epsilon / 100+\epsilon t \mathrm{R}) \gamma-10 .
\end{aligned}
$$


The reaction equation for $(\epsilon / 100+\epsilon t \mathrm{R}) \mathrm{Id}$ is

$$
\begin{aligned}
\frac{\partial}{\partial t}(\epsilon / 100+\epsilon t \mathrm{R}) & =\frac{k}{400 t^{3 / 4}}+\epsilon \mathrm{R}+2 \epsilon t|\mathrm{Ric}|^{2}+\frac{k t R}{4 t^{3 / 4}} \\
& \geq \frac{k}{500 t^{3 / 4}}+\epsilon \mathrm{R}+\epsilon t\left(\alpha^{2}+\beta^{2}+\gamma^{2}+\alpha \beta+\alpha \gamma+\beta \gamma\right),
\end{aligned}
$$

for $t \leq S\left(N, v_{0}, \sigma\right)$, in view of Claim (i). Combining these three inequalities we get

$$
\begin{aligned}
& \frac{\partial}{\partial t}\left(\alpha h+\left(\frac{\epsilon}{100}+\epsilon t \mathrm{R}\right)\right) \\
& \geq h \alpha^{2}+\left(h \alpha+\frac{\epsilon}{100}+\epsilon t \mathrm{R}\right) \gamma+\epsilon\left[\mathrm{R}-\gamma \frac{1}{100}\right] \\
& \quad+\epsilon t\left[-\mathrm{R} \gamma+\alpha^{2}+\beta^{2}+\gamma^{2}+\alpha \beta+\alpha \gamma+\beta \gamma\right]+\frac{k}{800 t^{3 / 4}} \\
& =h \alpha^{2}+\left(h \alpha+\frac{\epsilon}{100}+\epsilon t \mathrm{R}\right) \gamma+\epsilon\left[\mathrm{R}-\gamma \frac{1}{100}\right]+\epsilon t\left[\alpha^{2}+\beta^{2}+\alpha \beta\right]+\frac{k}{800 t^{3 / 4}} \\
& \geq h \alpha^{2}+\left(h \alpha+\frac{\epsilon}{100}+\epsilon t \mathrm{R}\right) \gamma+\epsilon\left[\mathrm{R}-\gamma \frac{1}{100}\right]+\frac{k}{800 t^{3 / 4}} .
\end{aligned}
$$

Assuming we have a first time and point $(y, s)$ where $Y(y, s)=0, y \in{ }^{t} B_{(1-\sigma / 5)}\left(x_{0}\right)$ then we must have $h(y, s)>0$ as we explained at the beginning of the proof of this Claim. At $(y, s)$ we have that $\alpha(y, s)=-(1 / h)(s \epsilon(s) \mathrm{R}(y, s)+\epsilon(s) / 100)<$ $(1 / h)\left(4 s \epsilon(s) \epsilon_{0}-\epsilon(s) / 100\right)<0$ (in view of Claim (i)) and hence the reaction equation of $Y=h \alpha+\epsilon t \mathrm{R}+\epsilon / 100$ may be estimated by

$$
\begin{aligned}
\frac{\partial}{\partial t}\left(\alpha h+\left(\frac{\epsilon}{100}+\epsilon t \mathrm{R}\right)\right) & \geq h \alpha^{2}+\left(h \alpha+\frac{\epsilon}{100}+\epsilon t \mathrm{R}\right) \gamma+\epsilon\left[\mathrm{R}-\gamma \frac{1}{100}\right]+\frac{k}{800 t^{3 / 4}} \\
& \geq h \alpha^{2}+\epsilon\left[\mathrm{R}-\gamma \frac{1}{100}\right]+\frac{k}{800 t^{3 / 4}},
\end{aligned}
$$

in view of the estimate (5-3), where we have used the fact that $Y(y, s)=0$. Then $[-\gamma / 100+\mathrm{R}]=[(\alpha+\beta) / 100+99 \mathrm{R} / 100] \geq[2 \alpha / 100+4 \mathrm{R} / 100+95 \mathrm{R} / 100] \geq$ $(2 / 100)[\alpha+2 R]-(95 / 100) \epsilon_{0} \geq-10 \epsilon_{0}$ in view of Claim (i) and (ii). Hence

$$
\frac{\partial}{\partial t} Y \geq h(y, s) \frac{\alpha^{2}}{2}+\frac{k}{801 t^{3 / 4}} .
$$

Now we examine the reaction diffusion equation. Using the estimate on the reaction equation above, we see that at time $(y, s)$ in direction $V$ where $Y(y, s)(V, V)=0$,

$$
\begin{aligned}
\frac{\partial}{\partial t} Y(V, V) \geq(\Delta Y)(y, s)(V, V)-2\left(g^{i j} \nabla_{j} h\right) & (y, s)\left(\nabla_{i} \mathcal{R}\right)(y, s)(V, V) \\
& +h(y, s) \frac{1}{2} \alpha^{2}(y, s)+\frac{k}{801 t^{3 / 4}} .
\end{aligned}
$$


We estimate the second term (the gradient term) of the right hand side of this inequality as follows:

$$
\begin{aligned}
& -2\left(g^{i j} \nabla_{j} h\right)(y, s)\left(\nabla_{i} \mathcal{R}\right)(y, s)(V, V) \\
& =-\frac{2}{h(y, s)} g^{i j} \nabla_{j} h \nabla_{i}(\mathcal{R} h)(y, s)(V, V)+2 \alpha(y, s) \frac{|\nabla h|^{2}}{h} \\
& =-\frac{2}{h(y, s)} g^{i j} \nabla_{j} h(y, s) \nabla_{i}\left[\mathcal{R} h+\frac{\epsilon}{100} \mathrm{Id}+\epsilon t \mathrm{R} \operatorname{Id}\right](y, s)(V, V) \\
& \quad+2 \alpha(y, s) \frac{|\nabla h|^{2}}{h}+\frac{2 \epsilon s}{h(y, s)} g^{i j}\left(\nabla_{i} \mathrm{R} \nabla_{j} h\right)(y, s)(V, V) \\
& =2 \alpha(y, s) \frac{|\nabla h|^{2}}{h}+\frac{2 \epsilon s}{h(y, s)} g^{i j}\left(\nabla_{i} \mathrm{R} \nabla_{j} h\right)(y, s)(V, V) \\
& \geq-\frac{1}{4} \alpha^{2}(y, s) h(y, s)-2 \frac{|\nabla h|^{4}}{h^{3}}(y, s)-\frac{2 \epsilon s}{h(y, s)}|\nabla \operatorname{Riem}|(y, s)|\nabla h|(y, s) \\
& \geq-\frac{1}{4} \alpha^{2}(y, s) h(y, s)-C(\sigma)-\frac{2 \epsilon s}{h(y, s)}|\nabla \operatorname{Riem}|(y, s)|\nabla h|(y, s),
\end{aligned}
$$

since $|\nabla \varphi|^{2} \leq \varphi^{3 / 2} C(\sigma)$. Using the estimates of Shi, see [9, Theorem 3.1], and the fact that $|\operatorname{Riem}|(x, t) \leq N / t$, we see that $|\nabla \operatorname{Riem}|^{2} \leq \widehat{c} N^{3} / t^{3}$ where $\widehat{c}=\widehat{c}(N, \sigma)$. We explain this in more detail. $x \in{ }^{t} B_{(1-\sigma / 5)}\left(x_{0}\right)$ implies ${ }^{t} B_{\sigma / 1000}(x) \subseteq{ }^{t} B_{1}\left(x_{0}\right)$ for the $x$ we are considering. We work in the ball ${ }^{t} B_{\sigma / 1000}(x)$, and we have $|\operatorname{Riem}(\cdot, t)| \leq \frac{N}{t}$ there. Scale so that $t=1$. Note that $|\operatorname{Riem}| \leq 2 N$ for $t \in(1 / 2,1]$ after scaling, so distances change in a controlled manner near time 1 . This allows one to find a parabolic region of the form ${ }^{g(1)} B_{r}(x) \times\left[-r^{2}, 1\right]$ for some $r=r(\sigma, N)>0$ close to one on which $\mid$ Riem $\mid \leq 2 N$. Now we may use the estimates of Shi, see [9, Theorem 3.1], at $t=1$, and then scale back to $t$ (this completes the explanation of the estimate $\mid \nabla$ Riem $\left.\left.\right|^{2} \leq \widehat{c} N^{3} / t^{3}\right)$. Using $\mid \nabla$ Riem $\left.\right|^{2} \leq \widehat{c} N^{3} / t^{3}$, we get

$$
\frac{2 \epsilon s}{h(y, s)}|\nabla \operatorname{Riem}|(y, s)|\nabla h|(y, s) \leq \frac{4 \widehat{c} N^{3 / 2} \epsilon_{0}}{h(y, s) s^{1 / 2}}|\nabla h|(y, s) .
$$

Notice that we must have $h(y, s) \geq \epsilon_{0} s / N 500$. If not, then

$$
\begin{aligned}
h \alpha+\left(\epsilon s \mathrm{R}+\frac{\epsilon}{100}\right) \text { Id } & \geq-h \mid \text { Riem } \mid-\frac{\epsilon_{0}}{400}+\frac{\epsilon_{0}}{200} \\
& \geq-h \mid \text { Riem } \mid+\frac{\epsilon_{0}}{400} \\
& \geq-\frac{N \epsilon_{0}}{N 500}+\frac{\epsilon_{0}}{400} \\
& >0,
\end{aligned}
$$


(at $(y, s))$ if $s \leq S\left(N, v_{0}, \sigma\right)$, which is a contradiction (here we have used again that $|\operatorname{Riem}(g(t))| \leq N / t$ and $\epsilon(s) s \mathrm{R} \geq-\epsilon_{0} / 400$ for $s \leq S\left(N, \sigma, v_{0}\right)$ small enough in view of Claim (i)). Hence

$$
\begin{aligned}
\frac{2 \epsilon s}{h(y, s)}|\nabla \operatorname{Riem}|(y, s)|\nabla h|(y, s) & \leq \frac{4 \widehat{c} N^{3 / 2} \epsilon_{0}}{h(y, s) s^{1 / 2}}|\nabla h|(y, s) \\
& \leq \frac{4 c \widehat{c} N^{3 / 2} \epsilon_{0} h^{3 / 4}}{h(y, s) s^{1 / 2}} \\
& =\frac{4 c \widehat{c} N^{3 / 2} \epsilon_{0}}{h^{1 / 4}(y, s) s^{1 / 2}} \\
& \leq \frac{4(500 N)^{1 / 4} c \widehat{c} N^{3 / 2} \epsilon_{0}}{s^{3 / 4} \epsilon_{0}^{1 / 4}} \\
& \leq \frac{\widehat{c} \epsilon_{0}^{3 / 4} c(500 N)^{2}}{s^{3 / 4}},
\end{aligned}
$$

where we have once again used that $|\nabla h|^{4} \leq C h^{3}$.

Substituting this inequality into (5-5), we obtain

$$
-2 g^{i j} \nabla_{j} h \nabla_{i} \mathcal{R}(y, s)(V, V) \geq-\frac{1}{4} \alpha^{2}(y, s) h(y, s)-C-\frac{\widehat{c} \epsilon_{0}^{3 / 4} c(500 N)^{2}}{s^{3 / 4}} .
$$

Substituting the inequality (5-6) into (5-4) we see that

$$
\frac{\partial}{\partial t} Y(y, s)(V, V)>(\Delta Y)(y, s)(V, V),
$$

if $k=k\left(N, \sigma, v_{0}\right)$ is chosen large enough. This contradicts $(y, s)$ being a first time and point where $Y$ is zero. This implies that $Y$ remains larger than zero for a well defined time interval $\left[0, S\left(N, v_{0}, \sigma\right)\right)$ as long as $t \leq S_{\max }$.

Using the definition of $\varphi$ (which is used in $h$ ) we see that

$$
\alpha \geq-\frac{2 \epsilon}{100}-2 \epsilon t \mathrm{R} \geq-4 \epsilon_{0} N>-1
$$

on ${ }^{t} B_{(1-\sigma)}\left(x_{0}\right)$ for $t \leq S\left(N, v_{0}, \sigma\right)$ as long as $t \leq S_{\max }$. Here we have used $|\mathrm{R}(\cdot, t)| t \leq N$.

That is, condition (a) will also not be violated for a well-defined time interval. That is $S_{\max } \geq S\left(n, N, v_{0}\right)>0$. This finishes the proof of Claim (iv) and the proof of the Theorem if one accepts Claim (v) (note that ( $\tilde{b})$ also holds).

Claim (v) If $d_{t}(\cdot)$ is not differentiable at $x \in M$ then we use the trick of E Calabi [1] as explained in the proof of Claim (iii) of the proof of Theorem 4.1. 
Hence we may argue with $\tilde{h}(y, s):=e^{-2 k_{0} s} \tilde{k}(y, s)$ everywhere above. If for example $(x, t)$ is a first time and point where $(h(\mathcal{R}+2 \mathrm{R} \mathrm{Id})+\epsilon \mathrm{Id})(V, V)=0$, then $(x, t)$ is a first time and point where $(\tilde{h}(\mathcal{R}+2 \mathrm{R} I \mathrm{~d})+\epsilon \mathrm{Id})(V, V)=0$, at least locally (see the proof of Claim (iii) of the proof of Theorem 4.1 for further details). Arguing as in the proof of Claim (iii) of the proof of Theorem 4.1, we see that such an $(x, t)$ cannot exist.

A similar argument holds for the tensor in Claim (iv) of this proof.

This finishes the proof of Claim (v) of this proof and the proof of the theorem.

Remark 5.3 Theorem 5.1 establishes Theorem 1.5 for the case $V=1 / 400 N, N \geq 1$. The case of general $V>0$ may be obtained as follows. Scale so that $r=1$. Now scale again, so that $\mathcal{R} \geq-1 / 400 N$ on ${ }^{0} B_{r}\left(x_{0}\right)$, where $r=\sqrt{V 400 N}$ (notice that we require $V>0$ to do this). We now have $\operatorname{vol}\left({ }^{0} B_{r}\left(x_{0}\right)\right) \geq \widehat{v}_{0}=\widehat{v}_{0}\left(V, N, v_{0}\right)>0$. Now repeat the proof of Theorem 5.1 with the following changes: replace $v_{0}$ by $\widehat{v}_{0}$, replace all balls ${ }^{t} B_{s}\left(x_{0}\right)$ that appear in the proof by ${ }^{t} B_{r s}\left(x_{0}\right)$, choose a cutoff function $\varphi$ from Proposition 3.3 with $\varphi(s)=1$ for all $s \leq r(1-\sigma / 6)$ and $\varphi(s)=0$ for all $s \geq r(1-\sigma / 7)$ in Claim (ii) respectively with $\varphi(s)=1$ for all $s \leq r(1-\sigma / 2)$ and $\varphi(s)=0$ for all $s \geq r(1-\sigma / 3)$ in Claim (iv). The proof then works without any further changes, except that the constants that occur now also depend on $V$ (this dependence also appears in the statement of Theorem 1.5).

\section{References}

[1] E Calabi, An extension of E Hopf's maximum principle with an application to Riemannian geometry, Duke Math. J. 25 (1958) 45-56 MR0092069

[2] H-D Cao, S-C Chu, B Chow, S-T Yau, Collected papers on Ricci flow, Series in Geometry and Topology 37, International Press, Somerville, MA (2003) MR2145154

[3] A Chau, L-F Tam, C Yu, Pseudolocality for the Ricci flow and applications, Canad. J. Math. 63 (2011) 55-85 MR2779131

[4] B-L Chen, Strong uniqueness of the Ricci flow, J. Differential Geom. 82 (2009) 363-382 MR2520796

[5] B-L Chen, G Xu, Z Zhang, Local pinching estimates in 3-dim Ricci flow arXiv: 1206.1814

[6] B Chow, S-C Chu, D Glickenstein, C Guenther, J Isenberg, T Ivey, D Knopf, P Lu, F Luo, L Ni, The Ricci flow: techniques and applications, Part III: Geometric-analytic aspects, Math. Surveys and Monographs 163, Amer. Math. Soc. (2010) MR2604955

[7] R S Hamilton, Four manifolds with positive curvature operator, J. Differential Geom. 24 (1986) 153-179 MR862046 
[8] RS Hamilton, A compactness property for solutions of the Ricci flow, Amer. J. Math. 117 (1995) 545-572 MR1333936

[9] RS Hamilton, The formation of singularities in the Ricci flow, from: "Surveys in differential geometry", (S-T Yau, editor), volume II, Int. Press, Cambridge, MA (1995) 7-136 MR1375255

[10] B Kleiner, J Lott, Notes on Perelman's papers, Geom. Topol. 12 (2008) 2587-2855 MR2460872

[11] P Lu, A local curvature bound in Ricci flow, Geom. Topol. 14 (2010) 1095-1110 MR2629901

[12] G Perelman, The entropy formula for the Ricci flow and its geometric applications (2002) arXiv:math/0211159

[13] F Schulze, M Simon, Expanding solitons with non-negative curvature operator coming out of cones arXiv:1008.1408

[14] M Simon, Ricci flow of almost non-negatively curved three manifolds, J. Reine Angew. Math. 630 (2009) 177-217 MR2526789

[15] M Simon, Ricci flow of non-collapsed three manifolds whose Ricci curvature is bounded from below, J. Reine Angew. Math. 662 (2012) 59-94 MR2876261

[16] Y Wang, Pseudolocality of the Ricci flow under integral bound of curvature, J. Geom. Anal. 23 (2013) 1-23 MR3010270

Institut für Analysis und Numerik (IAN), Universität Magdeburg

Universitätsplatz 2, D-39106 Magdeburg, Germany

miles.simon@ovgu.de

Proposed: John Lott

Seconded: Tobias Colding, Yasha Eliashberg
Received: 21 September 2012

Revised: 18 June 2013 